\title{
QUID bei Fleischerzeugnissen, die unter Verwendung von Fleisch verschiedener Tierarten hergestellt werden
}

\author{
Dr. H. Kirchhoff und S. Rieckhoff \\ Niedersächsischen Landesamtes für Verbraucherschutz und Lebensmittelsicherheit (LAVES), Lebensmittelinstitut Oldenburg, \\ Martin-Niemöller-Str. 2, 26133 Oldenburg
}

\section{Zusammenfassung/Erläuterung}

Bei einigen Geflügelwürsten, die unter Verwendung von Hühnerfleisch und Putenfleisch hergestellt wurden, sind die Anteile an Huhn und Pute zusammen angegeben (z. B. Geflügelfleischwurst; Zutaten: 70\% Geflügelfleisch (Hühnerfleisch, Putenfleisch)). Dies entspricht nicht dem Ergebnis der Abstimmungen des ALS und ALTS, nach dem die Anteile an Fleisch aller verwendeten Tierarten mit Ausnahme von Rind- und Schweinefleisch getrennt angegeben werden müssen.

Bei Berücksichtigung von § 8 Abs.1Nr.1der LMKV ergibt sich jedoch h. E. eine andere Schlussfolgerung: „Die Menge einer verwendeten Klasse oder vergleichbaren Gruppe von Zutaten (Gattung von Zutaten) ist anzugeben, wenn die Bezeichnung der Gattung von Zutaten in der Verkehrsbezeichnung genannt ist.“ Daraus folgt, dass beispielsweise bei Geflügelfleischwurst die Menge an Geflügelfleisch insgesamt angegeben werden kann, auch wenn mehrere Arten Hausgeflügel (Huhn, Pute, Gans, Ente) verarbeitet worden sind. Ähnliches dürfte auch für Erzeugnisse aus Wildfleisch gelten (z. B. kann bei einer Wildpastete, die aus Hirsch- und Wildschweinfleisch hergestellt wurde, die Summe an Hirsch- und Wildschweinfleisch angegeben werden).

\section{Beschluss (einstimmig)}

Sofern bei nicht expliziter Nennung einer Tierart in der Verkehrsbezeichnung lediglich ein Gattungsbegriff genannt wird (z.B. Wildsalami, Geflügelbierschinken), genügt die zusammengefasste Mengenangabe für die jeweilige Gattung von $\mathrm{Zu}-$ taten (z. B. X\% Wildfleisch bzw. X\% Geflügelfleisch) den rechtlichen Anforderungen zur Mengenkennzeichnung. 